\title{
The distribution of exocrine glands in Lepeophtheirus salmonis and Caligus elongatus (Copepoda: Caligidae)
}

\author{
S. Bell, J.E. Bron \& C. Sommerville \\ Institute of Aquaculture, University of Stirling, Stirling, Scotland FK9 4LA
}

Keywords: gland, sea lice, diaminobenzidine, peroxidase, Caligidae, Copepoda

\begin{abstract}
The morphology, function and distribution of exocrine glands of copepods have rarely been studied in detail and almost nothing is known about them in the sea lice species $L_{x}$ salmonis and $C$. elongatus. This study utilised a novel application of a light-microscopy staining technique to reveal a variety of glands in nauplius, copepodid, chalimus, preadult and adult stages. The stain, 3',3-diaminobenzidine tetrahydrochloride (DAB), applied to fresh material, differentiated a population of exocrine glands and enabled a study of their distribution. The stain was successful in highlighting the ducts and pores associated with the larger glands. The locations of gland sub-populations were conserved in all life-stages, although glands in the swimming legs were found to be better represented in mobile and freeswimming stages i.e. copepodids, preadults and adults. Glands associated with the mouth-tube were also located. Other, nonglandular, peroxidase-positive regions were also highlighted by the stain. These regions were found mainly in preadult and adult stages where they formed characteristic bi-symmetrical patterns on the cuticle of the dorsal surface. A study of some calanoid copepods suggested that peroxidase-positive glands are a feature peculiar to caligid copepods. This staining technique has proven useful for elucidating the ontogeny of gland populations in caligids.
\end{abstract}

\section{Contents}

Introduction

Material and methods

DAB

Light microscopy

Results

Lepeophtheirus salmonis

Naupliî

Copepodid

Chalimus

Preadults $\begin{array}{ll}\text { Adults } & 15 \\ \text { Caligus elongatus } & 16 \\ \text { Other species } & 17 \\ \text { Discussion } & 17 \\ \text { References } & 19\end{array}$

\section{Introduction}

Descriptions of glandular structures of copepods have been a feature of the literature for many decades (Richards, 1891; Yonge, 1932; Clarke, Conover, David, \& Nicol, 1962; Fahrenbach, 1962; Park, 1966; Mauchline, 1977; Briggs, 1978; Gharagozlou-van Ginneken, 1979; Arnaud, Brunet, \& Mazza, 1988a; Arnaud, Brunet, \& Mazza 1988b; Dumont \& Silva-Briano, 1997). Unfortunately the few detailed studies which have been undertaken serve only to highlight our lack of understanding of these structures, their secretions and their significance to the biology and ecology of these animals.

Despite the fact that many different types of glands have been located in various parts of the copepod body, few of them have had their secretions characterised and their biological significance determined conclusively. Those best studied include the glands of the buccal cavity (Boxshall, 1982; Arnaud et al., 1988ab; Zeni \& Zaffagnini, 1992; Vaupel Klein \& Koomen, 1994) the luminous glands of the Calanoida (Clarke et al., 1962; Herring, 1988; Bannister \& Herring, 1989) and the mucous glands of the capsule-dwelling copepods (Hicks \& Grahame, 1979). Glandular complexes in the urosome have been described previously by 
(Gharagozlou-van Ginneken, 1979) who suggested they produced chemicals involved in sexual activity. Fahrenbach (1962) and Boxshall (1982) also described glands in the urosomes of copepods but did not suggest a function for them. Glands in the thoracic limbs have also been widely reported (Fahrenbach, 1962; Park, 1966; Dumont \& SilvaBriano, 1997).

Other glands are commonly found in close association with the integument ("integumental glands") but their role is still the subject of debate. Dennell (1947) and Stevenson (1961) suggested that they might be involved in cuticle tanning, while Rybakov \& Dolmatov (1991) suggest a role in cuticle formation. Other functions such as predator deterrence (Pochon-Masson, Renaud-Mornant, \& Cals, 1975) immunity to host nematocysts (Briggs, 1978), cuticle anti-fouling (Boxshall, 1982; Bannister, 1993), drag reduction (Bannister, 1993) "secretiontrap' for water-borne chemical cues (HipeauJacquotte, 1987) and production of pheromones for mate attraction (Gharagozlou-van Ginneken, 1979) have all been suggested as functions of these glands. It is likely that there is variation in integumental gland products and functions between species which probably represent evolutionary changes necessitated by the particular environment in which the animal lives.

In contrast to the widely accepted idea that glands are actively involved in secretion, Chapman (1981) showed that the "dermal'glands of Neocalanus plumchrus were involved in the uptake of dissolved glucose from the surrounding sea water.

Previous work (Bron, 1993; Andrade-Salas, 1997) has shown that the immunohistochemical stain $3^{\prime}, 3-$ diaminobenzidine tetrahydrochloride (DAB) highlighted glandular regions in $L$, salmonis larvae and adults. DAB is a chromogenic compound used routinely in immunohistochemical analyses. It is often used coupled to antibodies, as a marker, to highlight areas of antibody binding. Used on its own however it reacts, in the presence of hydrogen peroxide $\left(\mathrm{H}_{2} \mathrm{O}_{2}\right)$ to produce a characteristic brown product which is insoluble in water, alcohol and xylene. This positive reaction to the DAB stain generally occurs with endogenous peroxidases but may also occur with catalase, cytochrome oxidase and possibly a number of other compounds.
Peroxidase enzymes have been reported to be involved in many different functions within animal tissues, including: free radical neutralisation (Salin \& Brown-Peterson, 1993), prostaglandin production (Bowman, Dillwith, \& Sauer, 1996) and iodine binding as part of protothyroid activity in tunicates (Fredriksson, Öfverholm, \& Ericson, 1988).

As part of a wider investigation into the functional significance of exocrine glands in $L$, salmonis we undertook a study to locate all the DAB-positive regions in all life-stages of that species and the related parasitic copepod Caligus elongatus. To determine whether DAB-positive regions are a widespread feature of the Copepoda, we stained several species of free-living copepod and also some other aquatic arthropods.

\section{Materials and methods}

\section{$D A B$}

One 10mg DAB tablet (Sigma D5905) was added to $15 \mathrm{ml}$ of Tris buffer to give a $0.66 \% \mathrm{w} / \mathrm{v}$ solution to which $12 \mu \mathrm{l}$ of $\mathrm{H}_{2} \mathrm{O}_{2}$ was added $(0.026 \%$ solution) to provide a substrate for the reaction. The reaction was halted by the addition of $70 \%$ ethanol.

Fresh specimens were added to the prepared DAB solution and left to stain at room temperature for a range of time periods. The optimal staining time was found to be: $\approx 15 \mathrm{~min}$ for nauplii and copepodids, $\approx 6 \mathrm{~h}$ for chalimus and $\approx 16 \mathrm{~h}$ for preadults and adults. After these periods had elapsed the reaction was halted by carefully pipetting off the stain solution and replacing it with $70 \%$ ethanol.

Chalimus, preadult and adult stages were stained soon after collection (i.e. not exceeding $48 \mathrm{~h}$ postremoval) to minimise any effects on gland activity that being removed from the salmon host may incur.

The same procedures were followed for nauplius, copepodid and adult specimens of the caligid copepod Caligus elongatus Nordmann, 1832. Adults of the free-living marine copepods Eurytemora affinis (Poppe, 1880) and Acartia tonsa Dana, 1849, the cladoceran Daphnia magna Straus, 1820, and 
larval stages of the anostracan Artemia salina (L.), and the palaemonid Macrobrachium rosenbergii (de Man) were also stained in the same way for comparison.

\section{Light microscopy}

Small larval stages (i.e. nauplius, copepodids and chalimus I and II) were examined in a cavity slide under a cover slip. Lactic acid (87.5\%) was used to aid in the clearing of the body tissues of larval stages, to enhance visualisation of gland structures without affecting the quality of staining, although this method could not be used as a permanent mountant (Huys \& Boxshall, 1991).

Observations were made using an Olympus BH2 compound microscope and photographs were taken using an Olympus C-35AD-2 camera.

\section{Results}

\section{Lepeophtheirus salmonis}

DAB was successful in highlighting gland structures in all life-stages of $L$. salmonis. In chalimus, preadult and adult stages there was considerable variability between specimens of the same sample with regard to their staining characteristics. Pictures of gland distribution in these stages were therefore constructed from multiple specimens. The DAB stain also picked up some other, non-glandular features. In both $L$. salmonis and $C$. elongatus retinula cells of the eye and parts of the cerebrum often stained moderately with $\mathrm{DAB}$, as did some of the body musculature, particularly the attachment points of the muscles to the cuticle. In specimens with damaged integuments it was common to see strong DAB-positive staining around the tears and cuts in the cuticle. It is not possible to say whether such staining is due to DAB-positive components in the exposed cuticle or whether this represents an active mechanism where DAB-positive products are aggregating at sites of injury.

\section{Nauplii}

Both the first (NI) and second (NII) nauplius larvae displayed the same general pattern of DABpositive staining, although some slight differences were evident. In addition to two large dorsal and ventral 'median' glands, both larval stages consistently displayed three pairs of distinct glandular regions arranged in a bilaterally symmetrical pattern (Fig. 1). These gland populations have been named in accordance with their location within the animal.

In addition to these glands, late NII larvae possessed up to 10 small DAB-positive areas, arranged in a symmetrical pattern, in the posterior half of the body between the lateral and posterior glands, position 'Th' in Fig. 1. These areas corresponded to the position of glands present in the thoracic limbs of the following copepodid stage. The posterior glanids exited via two small pores, one lying close to the ventral side of each balancer.

During the staining process some individual, unhatched eggs of $L$. salmonis were inadvertently stained along with the hatched nauplius stages. Examination of these eggs showed that pre-hatch nauplii had the same pattern of DAB-positive gland distribution as the NI stage larvae.

\section{Copepodid}

Copepodid larvae displayed a similar pattern of DAB-positive glands to that of the preceding naupliar stages (Fig. 2). The posterior, lateral and anteripr groups and the dorsal and ventral median glands all showed little apparent difference. In addition, there were 10 distinct glands in the thoracic limbs, four in the first pair of thoracic legs (th $\left.{ }_{1}\right)$ and six in the second $\left(\mathrm{th}_{11}\right)$, the precursors of which could be seen under the ventral cuticle of late NII larvae. Some smaller, less distinct regions of staining were also seen i.e. 5 spots around the eyes and 3 spots on the free thoracic segment.

The median glands overlay each other, both directly overlying the mouth-tube and were seen to have several ducts associated with them. Although it was difficult to distinguish the precise relationship between these glands and their ducts, some 

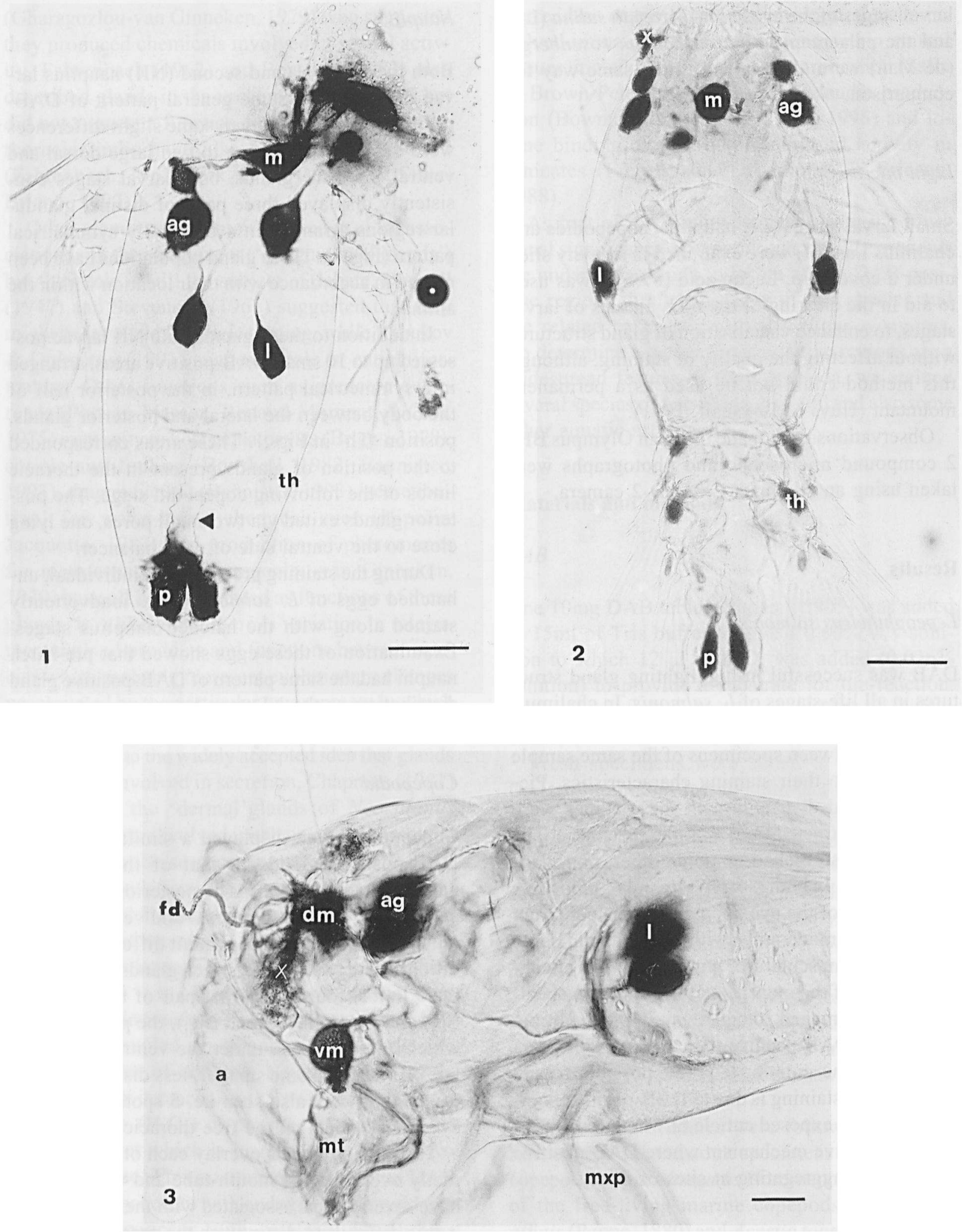
relationships could be visualised, as shown in Fig. 3. The ventral median gland was located at the base of the labrum and had a duct which passed into the labrum to exit on its anterior face approximately one third of the way down from the proximal end. A second DAB-positive duct appeared to lead from one of the median glands and passed in an anterodorsal direction to enter the developing filament. The duct stained clearly and extended to the distal tip of the undeveloped filament. The dorsal median gland had at least one duct arising from it which led to a pore is the dorsal cuticle immediately overlying the gland.

As the copepodid larvae have a functional gut, in contrast to the naupliar stage, it seems reasonable to re-name the posterior glands of the nauplius the 'perianal glands' at the copepodid stage. This fits the terminology of (Boxshall, 1982).

\section{Chalimus}

All stages of chalimus larvae appeared to possess all the DAB-positive glands located in the preceding larval stages, but during development from chalimus I to chalimus IV the distribution of glands changes. Chalimus larvae often exhibited variable staining which hindered the interpretation of a stagespecific pattern. The simplest pattern of gland distribution was found in chalimus I and II larvae: these still possessed the perianal, lateral and anterior glands of the previous stages, although the thoracic limb glands had become very indistinct. The anterior glands appeared to be composed of one/ two components in chalimus I and chalimus II, as in Fig. 4a, whereas it appeared to consist of two/ three components in the nauplius stage (see Fig. 1). This change may represent either a regression, or amalgamation, of the gland units. Other regions of staining, not apparent in the copepodid stage had developed in the chalimus larvae. A prominent feature of stained specimens was a complex of approximately six separate elements lying close to the cuticle of the dorsal surface immediately anterior to the eyes. This was termed the 'frontal gland complex' (FGC). This complex appeared to consist of some of the elements of the filamentproducing glands described by Bron, Sommerville, Jones \& Rae (1991). In particular, the A-glands described by Bron et al. (1991) stain strongly with $\mathrm{DAB}$, as did the three mucous glands which lie immediately in front of the eyes and extend anteriorly towards the A-glands. Other, smaller, associated structures also stained but their origin and significance could not be determined, although it is possible that they are part of the filament-producing complex.

Two pairs of symmetrical glands (or one pair of bi-lobed glands) surround the base of the mouthtube on the ventral surface and were termed 'circum-oral glands'. These glands are evident in Figs. $4 \mathrm{a}$ and $4 \mathrm{~b}$. It is possible that these are the ventral reaches of the mucous glands of the FGC. A similar pattern was seen in all three later chalimus larvae, but with a few, small differences. By chalimus III a pair of large glands close to the bases of the maxillae was apparent under the ventral cuticle. From each of these glands a fine duct ran in a medioventral direction and was presumed to exit close to the bases of the maxillules and maxillae. Although no exit pore could be detected in this region, there was evidence that a pore did lie in this area and that it was probably obscured by overlying appendages. This evidence was the presence of considerable quantities of DAB-positive material of a granular nature adhering to the cuticle close to the bases of the maxillules where the stained duct was seen to lead. These glands

\section{$\leftarrow$}

Fig. 1. DAB-stained second stage nauplius of $L$ salmonis showing glands and associated ducts e.g. the posterior gland ducts (arrowed). Note the anterior glands are composed of three connected regions. m median glands; ag anterior glands; 1 lateral glands; th thoracic limb glands precursor; p posterior glands. Scale bar $250 \mu \mathrm{m}$.

Fig. 2. Dorsal view of DAB-stained copepodid larva of L. salmonis. Note granular DAB secretion adhering to cuticle of antennules (x). m median glands; ag anterior glands; 1 lateral glands; th thoracic limb glands; p perianal glands. Scale bar $250 \mu \mathrm{m}$.

Fig. 3. Lateral view of anterior cephalothorax of DAB-stained copepodid larva of $L \times$ salmonis. Large regions of dispersed stained granules can be seen within the cephalothorax (x). ag anterior glands ; 1 lateral glands; mxp maxillipeds; mt mouth-tube; vm ventral median gland; a antenna; dm dorsal median gland; fd filament duct. Scale bar $100 \mu \mathrm{m}$. 


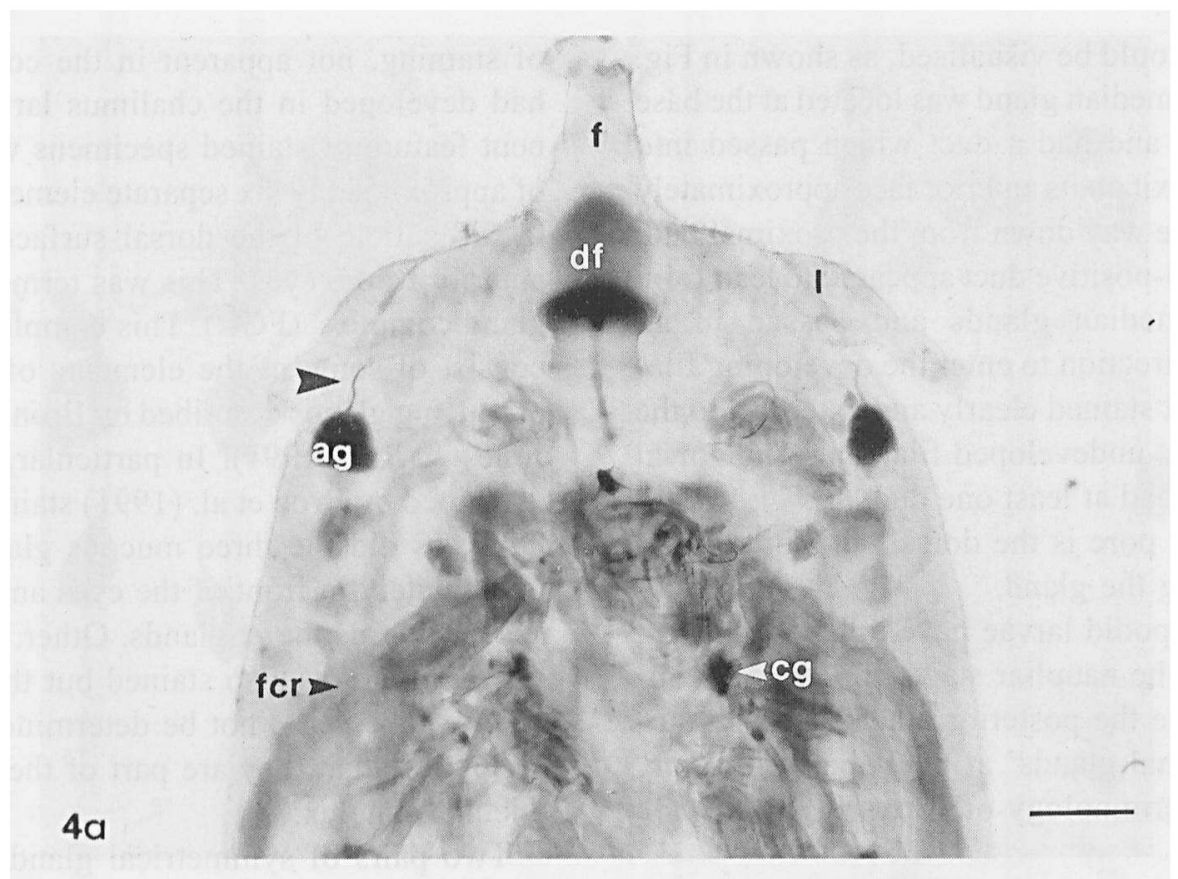

Fig. $4 a$. Ventral view of DAB-stained second stage chalimus larva of $L$. salmonis. Ducts are visible leading anteriorly from the anterior glands (arrowed). f existing filament; df developing filament; 1 antennule; ag anterior glands; fcr first cuticular ridge; $\mathrm{cg}$ circum-oral glands. Scale bar $200 \mu \mathrm{m}$.

are here termed the 'post-oral glands' until their homology with previously described glands has been determined. The possibilty that these glands correspond to the widely described maxillary glands found in other species of copepod and attributed with an excretory function, should be considered. In some specimens examined there was a large number of discrete DAB-positive granules/vesicles present within the body cavity. These regions are shown in Fig. 4b. These regions correspond to the cells of the mid-gut epithelium.

What were presumed to be the vestiges of the lateral glands could be discerned in the lateral regions of the cephalothorax, roughly level with the maxillipeds. These glands were very faint, consisting of loosley-packed grains. An anteriorly leading stained duct was associated with each of these glands.

In chalimus II some small, discrete regions of staining first appeared, lying close to the dorsal cuticle of the cephalothorax. There was an increase in their number during each successive chalimus stage, but no definite pattern characteristic of any one stage could be determined. There was no evidence to suggest that these regions of staining were glands per se as no secreted material was seen on the surface of the cuticle, and they shall therefore be referred to only as 'dorsal surface regions' (DSR).

\section{Preadults}

The gland distribution pattern of the preadult stages develops from that of the chalimus IV and showed an increase in complexity. There was also an increase in the number of DAB-positive glands from preadult I to preadult II in both sexes.

All of the main gland structures located in the chalimus larvae were also found in the preadult stages i.e. perianal, frontal gland complex, circumoral, postoral and dorsal surface regions, although no evidence of the lateral glands could be found. In the preadult stage there were two large groups of glands (or lobes of the same gland) present in the anterolateral regions of the cephalothorax. These groups, which were visible from both the dorsal and ventral surfaces, showed an increase in number of regions from preadult I to preadult II in both males and females. These gland complexes were given the name 'anterior gland complexes' (AGC) 


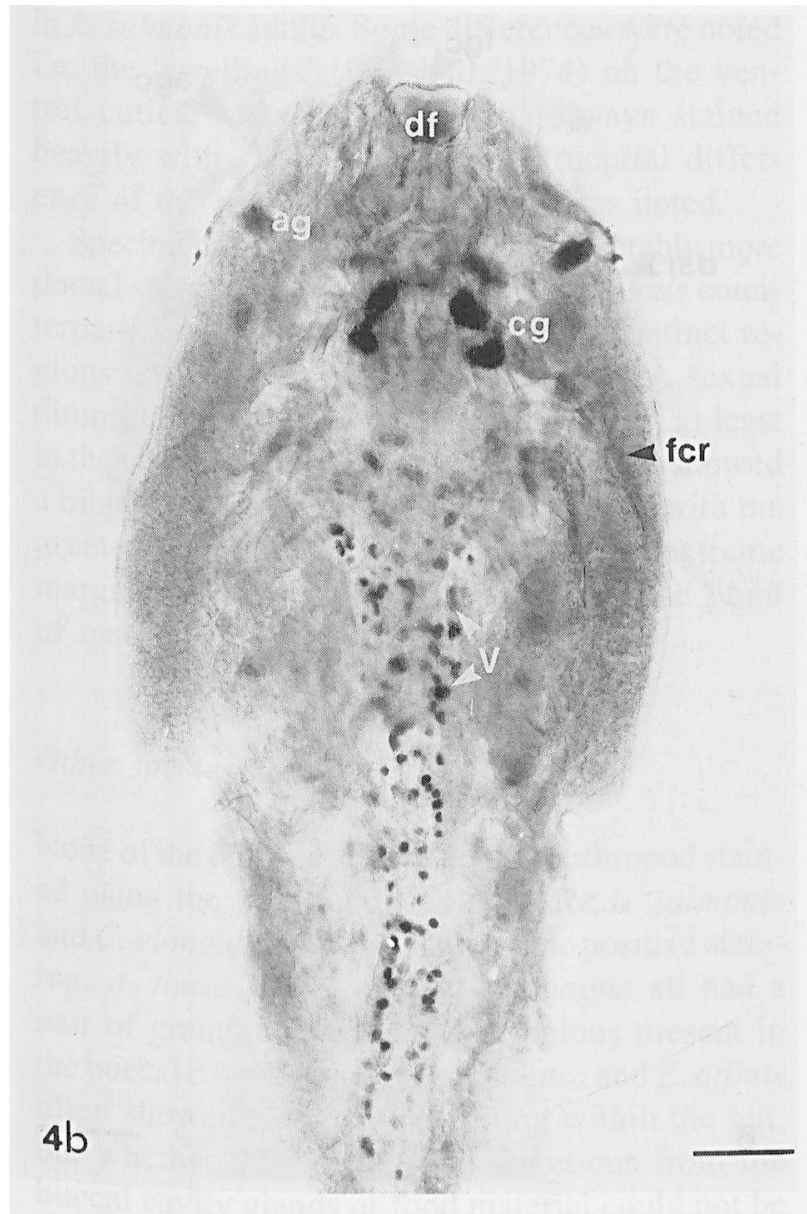

Fig. $4 b$. Ventral view of DAB-stained second chalimus ștage larva of $L$. salmonis. ag anterior glands; df developing filament; cg circum-oral glands; fcr first cuticular ridge; v vesicles lining gut. Scale bar $350 \mu \mathrm{m}$.

on account of the similarity of their location to the glands of the same name in earlier stages.

The postoral glands were again prominent and were situated close to the $\mathrm{AGC}$ in a region termed the 'lateral sinus'. When viewed from the ventral surface this lateral sinus was located immediately lateral to the maxillules and posterior to the post antennary processes. The posterior margin of this sinus was bounded by the 'first cuticular ridge' a ridge of thickened cuticle extending posterolaterally from close to the mouth-tube to the edge of the cephalothorax approximately half way along its length.

Circum-oral glands were present but their appearance differed from those in the chalimus stages: a large gland was evident at the base of the la- brum with two further glands lying immediately anterolateral to it, within the body cavity. These glands appeared to connect to the gland at the base of the labrum.

In common with the chalimus stages, the thoracic limbs showed very little staining. However, in contrast to the chalimus stages, the DSR were prolific and accounted for the bulk of DAB-positive staining found in preadults: $\approx 85$ in preadult $I$ females, $\approx 140$ in preadult II females, $\approx 56$ in preadult I males and $\approx 63$ in preadult II males. They were distributed in a bilaterally symmetrical pattern across the cephalothorax, and were especially concentrated around the margins. There were similar regions of staining on the dorsal and ventral surfaces of the genital segment. No secreted material was ever seen in association with any of these regions although quantities of DAB-positive material were commonly found on the setae of the caudal rami (particularly in preadult males) and was presumed to have originated in the perianal glands.

\section{Adults}

The greatest number of DAB-positive areas were found in adult specimens. The pattern of distribution was similar to that of the preadults but with some slight differences. The bulk of the staining could be accounted for by the DSR (Fig. 5).

Adult specimens retained all of the main glandular complexes described in the preceding stages. They had well developed perianal glands which consisted of one large region with up to six pairs of smaller associated regions. The secretions from these glands were often seen to coat the setae of the caudal rami. The circum-oral glands take the same form as those seen in the preadult stages, as did the frontal gland complex. The anterior gland complexes were large and composed of many elements, and together with the postoral glands filled the lateral sinus. The post-oral glands in adult stages appeared to have the same structural morphology seen in previous stages.

In adults DAB-positive glands become prominent in the first four pairs of thoracic legs, the setae of which were often seen to be covered in granu- 


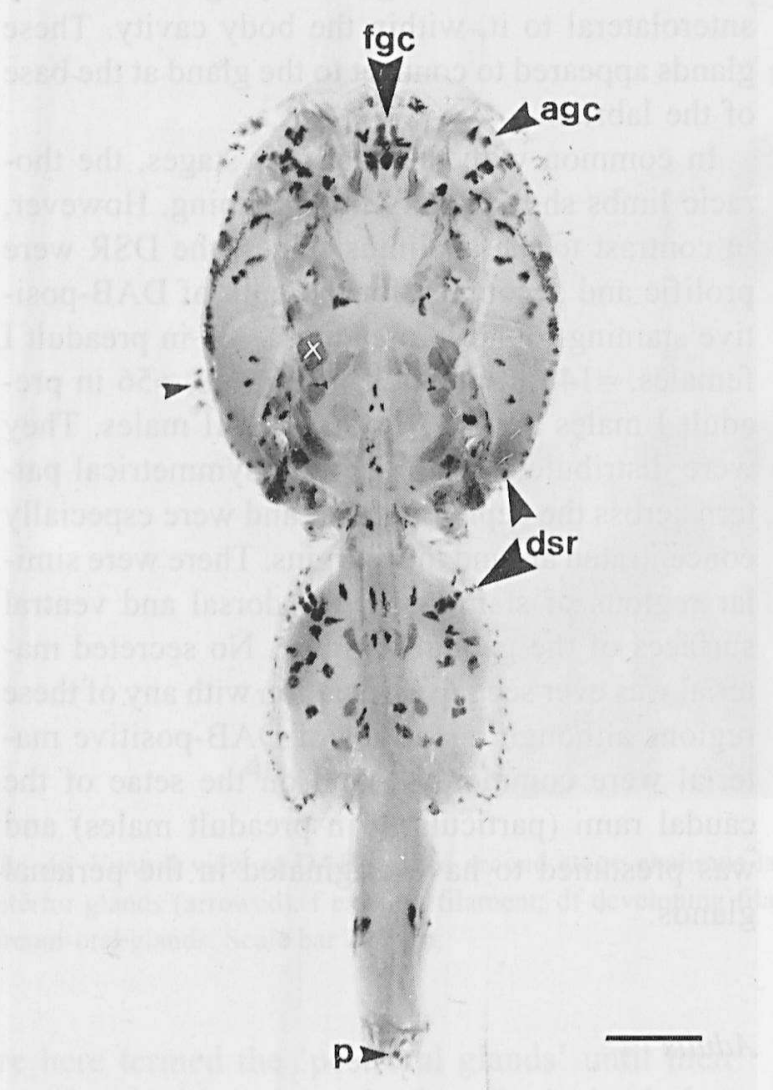

5

Fig. 5. Dorsal view of DAB-stained adult female $L$. salmonis. Note the DAB-positive material adhering to the marginal membrane of the cephalothorax (arrowed) and the regions of staining associated with muscle attachments $(x)$. p perianal glands; dsr dorsal surface regions; agc anterior gland complex; fgc frontal gland complex. Scale bar $1 \mathrm{~mm}$.

lar stained material. The staining of these glands was heavy and consistent between specimens.

The DSR at this stage, numbered at least 140 in females and 120 in males. The DSR were the most prominent feature of the stained animals. There was an obvious bilateral symmetry to the distribution of these areas, the greatest number of which were located around the margins of the cephalothorax. There appeared to be a sexual dimorphism with regard to DSR distribution, which was especially pronounced in the genital segment.

The marginal membrane often had DAB-positive material adhering to it, although it was not

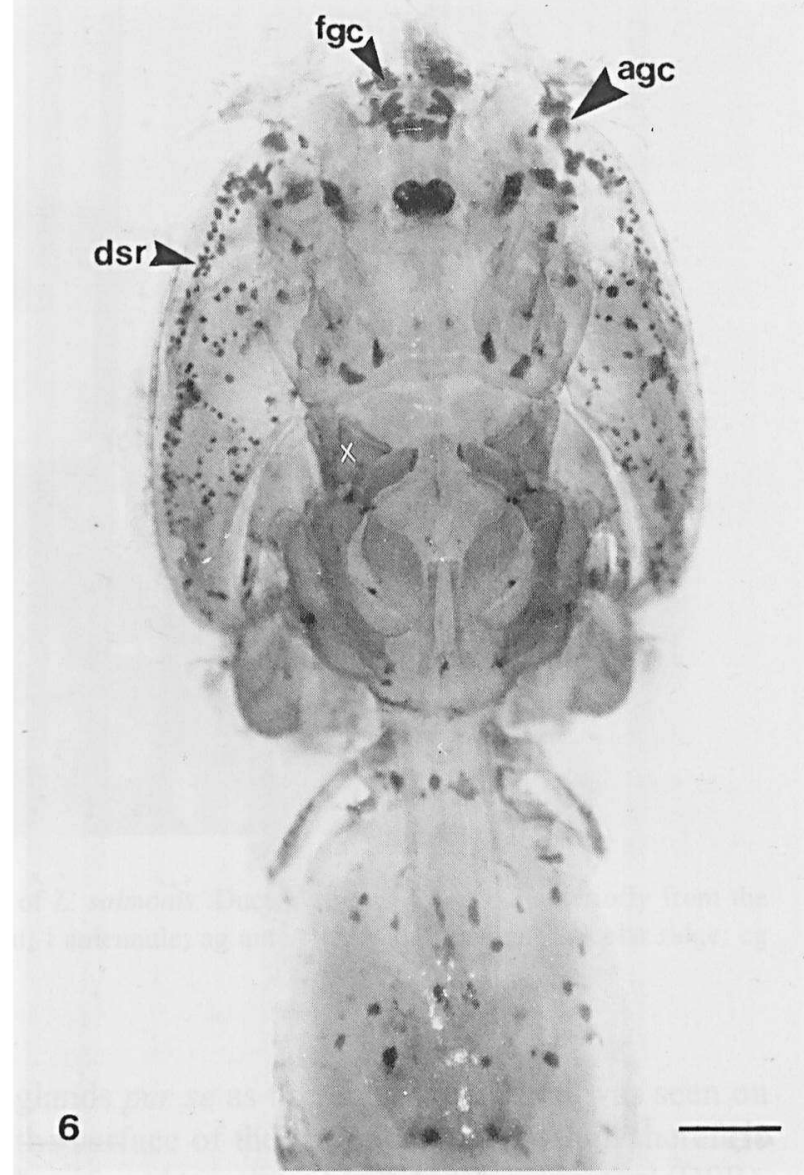

Fig. 6. Dorsal view of DAB-stained adult female C. elongatus. Note stained muscle attachment points on dorsal cuticle $(\mathrm{x})$. dsr dorsal surface regions; agc anterior gland complex; fgc frontal gland complex. Scale bar $500 \mu \mathrm{m}$.

clear whether this was secreted material, stained epibionts adhering to it, or regions of damaged integument.

\section{Caligus elongatus}

The nauplius and copepodid larvae of $C$. elongatus showed the same pattern of DAB-positive gland distribution found in the corresponding stages of L. salmonis.

Both adult male and female $C$. elongatus possessed all of the main DAB-positive regions seen 
in L. salmonis adults. Some differences were noted i.e. the 'swellings' (Boxshall, 1974) on the ventral cuticle between th ${ }_{1}$ and $\mathrm{th}_{\mathrm{II}}$ always stained heavily with DAB and a slight structural difference of the frontal gland complex was noted.

Specimens of C. elongatus had considerably more dorsal surface regions than their $L$. salmonis counterparts with adult males having $\approx \mathbf{2 8 0}$ distinct regions while females had $\approx 160$ (Fig. 6). A sexual dimorphism of these regions was evident, at least in the genital segment. These regions again showed a bilateral symmetry in their distribution, with the greatest proportion found around the extreme margins of the cephalothorax, close to the point of marginal membrane attachment.

\section{Other species}

None of the other species of aquatic arthropod stained using the technique employed for $L$. salmonis and $C$. elongatus showed comparable positive staining. A. tonsa, $E_{x}$ affinis and $D$. magna all had a pair of granular DAB-positive regions present in the buccal cavity region. Both $A$. tonsa and $E$. affinis often showed a region of staining within the gut, but whether this represented secretions from the buccal cavity glands or food material could not be determined.

The egg strings of female $E$. affinis were generally the only other regions of the body to stain with DAB. Specimens of $A$, salina, and $M$. rosenbergii showed no definite staining, although a slight darkening of the general body tissues was often seen,

\section{Discussion}

The DAB stain has proven useful as a tool for highlighting a population of exocrine glands in $L$. salmonis and $C$. elongatus. Whilst the present study has not attempted to determine the identity of products giving a positive reaction to DAB, this will be addressed in future. This work has shown that DAB-positive reactants are present in both species of sea lice at all life-stages, including prehatch nauplii.

Several of the glands found in this study have been reported previously. Bron (1993) first described DAB-positive glands in the thoracic limbs of $L$. salmonis copepodids and also located glands which appear to correspond to the circum-oral glands seen in chalimus larvae (Bron, Sommerville, \& Rae, 1993). The structure of the filament-producing glands in $L$. salmonis chalimus larvae has also been previously investigated (Bron et al. 1991; Bron, 1993) and superficially, components of the frontal gland complex found in the present study resemble some of those glands. Further analysis using histological/histochemical techniques will be required to determine the homologies of these glands. The fact that some of the filament-producing glands found by (Bron et al., 1991) stained positively with stains other than DAB, suggests a multi-component composition to the gland product and it is likely that many of the glands stained may have multiple functional components. Many exocrine secretions of copepods have a mucous component (Hicks and Grahame, 1979) and, whilst it has been demonstrated that sulphated mucopoly-saccharides may stain positively with DAB, Alcian Blue ( $\mathrm{pH} 1.0$ and 2.5) staining failed to demonstrate their presence in the exocrine glands of $L$. salmonis.

The glands in the thoracic limbs may be important with regard to the functioning of the limbs since they stain strongly only in the copepodid where swimming ability is important and in adults where swimming may similarly be important and where there are no further moults to renew the limbs and their setae. They are conspicuous by their greatly reduced appearance in the chalimus stages where limb function is relatively less important i.e. when filament-attached, and in preadult stages where there is a relatively short period between moults. As it was common to find large quantities of DAB-positive material adhering to the limb setae it may be that the secretion acts in some way to either maintain, or improve the function of those setae. This supports the suggestions of previous researchers who proposed cuticle tanning (Stevenson, '1961), anti-fouling (Boxshall, 1982; Bannister, 1993) and hydrophobic surfactants (Brunet, Cuoc, Arnaud, \& Mazza, 1991) as possible actions of gland products. It has been shown (Gresty \& Warren, 1993; Dumontet et al., 1996) that copepods frequently have epibiotic organisms attached to their cuticle and it is possible, there- 
fore, that secretions from the glands may be involved in killing or preventing the establishment of such organisms on the cuticle surface, a phenomenon found in corixid beetles where gland secretions prevent bacterial growth on the hairs of the physical gill (Kovac \& Maschwitz, 1991). This hypothesis does not account for why these regions appear to be unique to caligids, unless the environment in which they live is more likely to expose them to such fouling organisms.

Peroxidases have been shown to be present in red blood cells, hepatocytes, central nervous system neurons and oestrogen-sensitive tissues (Rainbow 1996) and are also known to be fundamental to the process of prostaglandin production from arachidonic acid (Bowman et al., 1996). Peroxidase enzymes and catalase have also been shown to be important neutralisers of potentially damaging free radicals, which result following tissue damage and are formed as by-products of metabolism and also from the interaction of U.V with natural organic matter. Peroxidase activity has also been show in tunicates where it is believed to be involved in iodination processes fundamental to protothyroid activity (Fredriksson et al., 1988).

The only DAB-positive glandular regions seen in free-living species are in the buccal cavity area. Whilst the function of these glands is unknown it may be suggested that peroxidases are being produced here to assist in the elimination of free radicals produced as a side-effect of feeding activity, a phenomenon witnessed in caterpillars (Felton \& Duffey, 1991). If such products are not quickly neutralised they might otherwise damage the gut epithelium. Glands in the buccal cavity region of free-living marine copepods have been well documented (Boxshall, 1982; Arnaud et al., 1988ab; Vaupel Klein \& Koomen, 1994) and L. salmonis and $C$. elongatus also have DAB-positive glands associated with their mouth-parts which may function in a similar manner, although it appears that at least one of them exits via a pore on the outside of the mouth-tube and it is therefore unclear how the secretion would interact with the food material.

It is possible that sea lice will be exposed to free-radicals, both as a result of host tissue damage, and following a host immune response which includes chemicals released at sites of tissue injury. The peroxidase compounds found in sea lice may be involved in protecting the parasite from the deleterious effects of such compounds. Prostaglandins, which are synthesized in a process involving peroxidase enzymes, have been shown to be involved in host inflammatory response modulation. Peroxidases and prostaglandins have been isolated in species of tick with unusually long hostattachment periods (Bowman et al., 1996). It seems reasonable to suppose that sea lice species may have developed methods to maintain a benevolent environment for themselves and this should be considered as a possible function of these glands. As very few DAB-positive regions were present in free-living species, as compared to the two parasitic species examined here, the DAB stain may be picking out features which represent specific developments associated with the parasitic mode of life.

It is probable that the glands highlighted by this stain do not all have the same function. In most cases the stain highlighted secreted material and we can presume that it is not gland ultrastructural elements which are being stained, but rather the secretion itself which shows the positive reaction. This is not however, true for the DSR where no associated ducts or secreted material have been observed. The possibility that the stain here is highlighting ultrastructural or neurological features will have to be addressed using other staining techniques and TEM. The results of a preliminary SEM examination suggest that the DSR may be associated with cuticular sensillae, adding further doubt to the idea that they are exocrine glands.

In summary, the DAB-positive gland distribution of $L$. salmonis had a consistent pattern which could be specifically identified at each life-stage, with the exception of chalimus larvae where staining difficulties hindered interpretation. The development of the fundamental gland groups could be followed through several life-stages i.e. perianal, lateral and anterior from nauplius to chalimus, while the perianal glands (and possibly the anterior gland complexes) were retained from nauplius to the adult. The median glands could be followed from nauplius to copepodid and may possibly be represented by the circum-oral and frontal gland complexes in 
later stages. We can presume that these glands have a fundamental role to play in the biology of these species. Histological evidence is being sought to determine the physical characteristics of the glands and their relationship with other organs and tissues in the body.

Bron (1993) and Bron et al. (1993) have provided evidence of the existence of further glands in $L$. salmonis which are not DAB-positive suggesting the DAB stain does not highlight all of the glands present in this species.

\section{References}

Andrade-Salas O. 1997. Feeding and digestion in the ectoparasitic copepod Lepeophtheirus salmonis (Kroyer, 1837). Unpublished doctoral dissertation, University of Stirling.

Arnaud J, Brunet M, Mazza J. 1988a. Labral glands in Centropages typicus (Copepoda, Calanoida). I. Sites of synthesis. $J$. Morphol. 197: 21-32.

Arnaud J, Brunet M, Mazza J. 1988b. The labral glands in Centropages typicus (Copepoda, Calanoida). II. Sites of secretory release. J. Morphol. 197: 209-219.

Bannister NJ. 1993. Distribution and structure of sub-cuticular glands in the copepod Temora longicornis. J. Mar. Biol. Assoc. U.K. 73: 97-107.

Bannister NJ, Herring PJ. 1989. Distribution and structure of luminous cells in four marine copepods. $J_{x}$ Mar. Biol. Assoc. U.K. 69: 523-533.

Bowman AS, Dillwith JW, Sauer JR. 1996. Tick salivary prostaglandins: presence, origin and significance. Parasilology Today 12: 388-396.

Boxshall GA. 1974. Lepeopththeirus pectoralis (O.F. Mäller,1776): a description, review and some comparisons with the genus Caligus Müller. J. Nat. Hist. 8: 445468.

Boxshall GA. 1982. On the anatomy of the misophrioid copepods, with special reference to Bentomisophria palliata Sars. Phil. Trans. Roy, Soc. London, B 297: 125-181.

Briggs RP. 1978. Structure of the integument in Paranthessius anemoniae Claus, a copepod associate of the Snakelocks anemone Anemonia sulcata (Pennant). J. Morphol. 156: 293-316.

Bron JE. 1993. A study of the biology.and behaviour of the copepodid larvae of the salmon louse Lepeophtheirus salmonis (Krøyer, 1837) (Copepoda: Caligidae). Unpublished doctoral dissertation, University of Stirling.

Bron JE, Sommerville C, Jones M, Rae GH. 1991. The settlement and attachment of early stages of the salmon louse, Lepeophtheirus salmonis (Copepoda: Caligidae) on the salmon host, Salmo salar, J. Zool. 224: 201-212.

Bron JE, Sommerville C, Rae GH. 1993. Aspects of the behaviour of copepodid larvae of the salmon louse (Krøyer, 1837). Boxshall GA, Defaye D, eds. Pathogens of Wild and Farmed Fish: Sea Lice. Chichester: Ellis Horwood, 125-142

Brunet M, Cuoc C, Arnaud J, Mazza J. 1991. Tegumental glands in a copepod Hemidiaptomus ingens: structural, ultrastructural and cytochemical aspects. Tissue and Cell 23: 733-743.

Bussolati G. 1971. Histochemical demonstration of sulfate groups by means of diaminobenzidine. Histochem. $J_{x} 3$ : 445-449.

Chapman PM. 1981. Evidence for dissolved glucose uptake from seawater by Neocalanus plumchrus (Arthropoda, Copepoda). Can. J. Zool. 59: 1618-1621.

Clarke GL, Conover RJ, David CN, Nicol JAC. 1962. Comparative studies of luminescence in copepods and other pelagic marine animals. J. Mar. Biol. Assoc. U.K. 42: 541-564.

Dennell R. 1947. The occurrence and significance of phenolic hardening in the newly formed cuticle of Crustacea. Proc, Roy. Soc. B 134: 485-503.

Dumont HJ, Silva-Briano M. 1997. Sensory and glandular equipment of the trunk limbs of the Chydoridae and Macrothricidae (Crustacea: Anomopoda). Hydrobiologia 360: 33-46.

Dumontet S, Krovacek K, Baloda SB, Grottoli R, Pasquale V, Vanucei S. 1996. Ecological relationship between Aeromonas and Vibrio spp and planktonic copepods in the coastal marine environment in southern Italy. Comp. Immunol. Microbiol. Infect. Dis. 19: 245-254.

Fahrenbach WH. 1962. The biology of a harpacticoid copepod. La Cellule 62: 303-376.

Felton GW, Duffey SS. 1991. Protective action of midgut catalase in lepidopteran larvae against oxidative plant defenses. J. Chem. Ecol, 17: 1715-1732.

Fredriksson G, Överholm T, Ericson LE. 1988. Iodine binding and peroxidase activity in the endostyle of Salpa fusiformis, Thalia democratica, Dolioletta gegenbauri and Doliolum nationalis (Tunicata, Thaliacea). Cell and Tissue Res. 253: 403-411.

Gharagozlou-van Ginneken ID. 1979. Etude ultrastructurale et cytochimique de l'activité temporaire des glandes tégumentaires d'un Crustacé Copépode. Ann. Scí. Nat. Zool. Paris 13: 205-212.

Gresty KA, Warren A. 1993. Incidence of ciliate epibionts on Lepeophtheirus salmonis from salmon in Japan and Scotland: a scanning electron microscopic study. Boxshall GA, Defaye D, eds. Pathogens of Wild and Farmed Fish: Sea Lice. Chichester: Ellis Horwood, 356-363.

Herring PJ. 1988. Copepod luminescence. In: Boxshall GA, Schminke HK, eds. Biology of Copepods. Hydrobiologia 167/168: $183-195$

Hicks GRF, Grahame J. 1979. Mucus production and its role in the feeding behaviour of Diarthrodes nobilis (Copepoda: Harpacticoida). J. Mar. Biol. Assoc. U.K. 50: 321330.

Hipeau-Jacquotte R. 1987. Ultrastructure and presumed function of the pleural dermal glands in the atypical male of the parasitic copepod Pachypygus gibber (Crustacea: Notodelphyidae). J. Crust. Biol. 7: 60-70. 
Huys R, Boxshall GA, 1991. Copepod Evolution. London Ray Society.

Kovac D, Maschwitz U. 1991. The function of the metathoracic scent gland in corixid bugs (Hemiptera, Corixidae): Secretion grooming on the water surface. $J_{x}$ Nat. Hist. 25: 331-340.

Mauchline J. 1977. The integumental sensilla and glands of pelagic crustacea, J. Mar, Biol, Assoc. U.K. 57: 973-994.

Park TS. 1966. The biology of a calanoíd copepod, Epilabidocera amphitrites McMurrish. La Cellule 66: 129-251.

Pochon-Masson J, Renaud-Mornant J, Cals P. 1975. Contribution à la connaissance des glandes tégumentaíres mètameriques $\mathrm{d}^{\text {'un }}$ crustacé meiobenthique interstitiel (Crustacea: Mystacocarida). Cytologie structurale et infrastructurale. Arch, Zool, Exp. Gen. 116: 123-146.

Rainbow RD. 1996. Immunohistochemistry. Woods AE, Ellis RC, eds. Laboratory Histopathology. A Complete Reference (Second ed.). Churchill Livingstone.

Richards J. 1891. Recherches sur le système glandulaire et sur le système nerveux des Copèpodes libres d'eau douce suivies d'une révision des espèces de ce groupe quì vivent en France. Ann. Sci. Nat. B 12: 113-260.

Rybakov AV, Dolmatov IY. 1991. Internal structure of the parasitic copepod Cucumaricola curvatus (Copepoda, Cucumaricolidae). Zoologicheskiy Zhurnal 70: 44-54.

Salin ML, Brown-Peterson NJ. 1993. Dealing with active oxygen intermediates: A halophilic perspective. Experientia 49: 523-529.

Stevenson JR. 1961. Polyphenol oxidase in the tegumental glands in relation to the molting cycle of the isopod crustacean Armadillidium vulgare. Biol. Bull. (Woods Hole) 121: 554-560.

Vaupel Klein JC van, Koomen P. 1994. The possible origins of mucus jets used for immobilizing prey in species of Euchirella (Copepoda, Calanoida, Aetideidae). 1. Theoretical considerations in relation to swimming and feeding behaviour. Crustaceana 66: 184-203.

Yonge CM. 1932. On the nature and permeability of chitin. 1. The chitin lining of the foregut of decapod crustacea and the function of the tegumental glands. Proc. Roy. Soc. B 111: 298-329.

Zeni C, Zaffagnini F. 1992. Labral glands of Leptestheira dahalacensis (Branchiopoda: Spinicaudata): An ultrastructural study, J. Crust. Biol. 12: 661-676.

Received: 4 December 1998 